\title{
Research on Fitness Gymnastics Courses in Private Higher Learning Institutions in Changchun
}

\author{
Landong $\mathrm{Xu}^{1, \mathrm{a}}$ \\ ${ }^{1}$ Changchun Guanghua University, Changchun, Jilin Province, China \\ a357951327@qq.com
}

Keywords: Changchun; private higher learning institutions; fitness gymnastics courses.

\begin{abstract}
This paper chooses 40 teachers and 400 students from 4 private higher learning institutions in Changchun as research objects, and uses research methods of documentary analysis, questionnaire survey and mathematical statistics to study the fitness gymnastics courses in these schools, in order to determine the research directions of fitness gymnastics courses. Then, countermeasures for optimizing gymnastics courses are put forward to theoretically guide the development of fitness gymnastics courses in private higher institutions in Changchun, and to provide references for promoting the development of fitness gymnastics courses in the physical education of private higher institutions.
\end{abstract}

\section{Introduction}

School physical education is the foundation of national fitness program and the plans of winning honor of Olympic Games. It is an important part of school education, and an important link in cultivating high-quality talents. How to cultivate high-quality talents who can meet the requirements of modern society is a serious challenge facing by schools all over the world. As an important part of school education, higher education plays a crucial role in improving national quality.

Gymnastics is an important part of college physical education in our country. It is important in realizing the goal of school physical education and improving the sports functions of universities. Fitness gymnastics is the extension of competitive gymnastics. It keeps up with times, and is rich in content. Fitness gymnastics pays attention to the cultivation of students' physical beauty and tastes, improves their coordination and aesthetic abilities, and helps them to learn skills and methods of body building. Fitness gymnastics injects vigor and vitality into campus, and provides a great help to physical fitness activities in society. The emergence of fitness gymnastics meets the requirements of social development. Through the study of fitness gymnastics, gymnastics can play a more important role in private higher institutions. This paper also provides theoretical guidance the relevant departments to further strengthen the construction of fitness gymnastics course system.

\section{Research Objects and Research Methods}

Research objects. Research objects of this paper are four higher learning institutions in Changchun: Changchun Guanghua University, Changchun Sci-Tech University, Changchun Architecture and Civilengineering College, and Changchun University of Finance and Economics

Research methods. Documentary analysis. According to the requirements of this subject, the author collected data from periodicals including Journals Database, core periodicals on sports and education, academic journals of physical education institutes and departments, and other publications in China. Relevant books, newspapers and legal documents issued by the Party Central Committee, the State Council, and the Ministry of Education are used as reference, too. This paper also refers to foreign language materials and relevant information which can be access from the Internet. Then the data is analyzed to provide theoretical guidance and research methods for this study.

Questionnaire survey. In order to better promote the development of fitness gymnastics courses in private higher learning institutions in Changchun, the author designed and issued 40 copies of Research on Fitness Gymnastics Cources in Private Higher Learning Institutions in Changchun- 
Teachers' Questionnaire and 400 copies of Research on Fitness Gymnastics Courses in Private Higher Learning Institutions in Changchun-Students' Questionnaire. 440 copies of questionnaire were collected, including 430 effective questionnaires. The recovery rate was $100 \%$; the efficiency rate was $97.7 \%$. Thus, the questionnaires have relatively good representativeness.

Interviews with experts. After collecting data, we consulted experts from Jilin Sport University and other private higher learning institutions through telephone calls, internet phone calls and interviews. We listened to their suggestions and ideas on the feasibility of fitness gymnastics courses in private higher learning institutions in Changchun.

\section{Results and Analysis}

The definition of fitness gymnastics. In general, we use the "genus concept plus specific difference" method to define concepts. Firstly, the genus concept of "fitness gymnastics" is studied. "Gymnastics" is a sports item which needs athletes to perform single, assembled or a complete set of movements on equipments with bare hands or holding light appliances. These movements have different difficulty levels and types; they aim to excavate people's potential, and express players' abilities to control their bodies. Meanwhile, gymnastics is a kind of sport which needs artistic presentation.

The definition of "fitness gymnastics" in this paper goes as follows. Fitness gymnastics does not belong to competitive gymnastics. It uses the general principles and methods of gymnastics, but it is easier to popularize and develop. The aims of fitness gymnastics are body building, improving people's physical health, mental conditions, cultural levels and artistic qualities. Fitness gymnastics covers mat exercises, Five Animal Exercises, aerobics, physical training, jumping and other activities. It is rich in content and organically combines art with sport. Thus, fitness gymnastics can meet college students' requirements of pursuing new and unique things, and can effectively improve students' physical conditions, strengthen their cardiopulmonary functions, and enhance the flexibility of their central nervous systems. All in all, the sport can promote the balanced development of students' bodies.

Content setting in fitness gymnastics classes. Fitness gymnastics courses should adhere to the guiding ideology of "health first", and pursue the enhancement of students physical qualities. In order to cultivate students' habits of taking exercise, teaching materials with high strong practicality and low difficulty should be used. Difficult and dangerous movements should be deleted; basic skills which are suit for students' physical and psychological characteristics should be introduced to enhance students' physical qualities. Sports skills should be integrated with theoretical ideas to improve students' cognitive levels. For example, rotating movements is good to improve the stability of central nervous system, and plays an important role in cultivating students' sensitivity and coordination abilities. Handstand is conducive to improve the regulatory function of nervous system and endocrine system. The movement reallocates blood in abdomen and chest, enhances memory ability and can effectively deals with mental disorder like addiction, depression and restlessness.

Teaching content should adhere closely to the goals of fitness gymnastics courses, meet the requirements of quality education and consider the needs of social development. Optimization should be applied to select more reasonable content and construct new curriculum system. The traditional curriculum content structure of competitive sports, especially the structure of sports skills, should be transformed into a teaching system which pays attention to the practical significance of sports culture, such as body building and ability training, from the perspective of education, and the potential significance of teaching materials, including human's socialization, personality cultivation and the role of emotion. The outline of fitness gymnastics courses are shown in table 1. 
Table 1: Teaching content and period distribution of fitness gymnastics course

\begin{tabular}{llc}
\hline & \multicolumn{1}{c}{$\begin{array}{c}\text { Teaching content } \\
\text { and reaching method }\end{array}$} & Teaching period \\
Theory Course & $\begin{array}{l}\text { 1. Methods of exercise in fitness gymnastics } \\
\text { 2. Functions of fitness gymnastics } \\
\text { in national fitness program }\end{array}$ & 2 \\
\hline & 1. Basic gymnastics & \\
Practical & 2. Mat exercises & 26 \\
Course & 3. Figure training & \\
& 4. Jumping & \\
\hline 5. Five Animal Exercises & 2 \\
Exams & 1. Theory exam & 2 \\
\hline 2. Practical exam & 3. Ability exam & 2 \\
\hline
\end{tabular}

Explanations for the teaching content of fitness gymnastics courses go as follows. (1) The teaching content and period distribution are made based on the basic conditions of students in private higher learning institutions. It is a new course created on the basis of gymnastics, and its aim is to cover the shortages of gymnastics courses. (2) On the basis of summarizing experiences of fitness gymnastics courses in Changchun Guanghua University, we select teaching content with good results and remove inappropriate materials. (3) A lot of teaching contents in this course are same as gymnastics courses, but the teaching methods of these two kinds of courses are totally different. Fitness gymnastics course pays more attention to practicability.

Students' attitudes towards fitness gymnastics. Interest is the best teacher. We instigate students' purposes of taking fitness gymnastics classes. The results can be seen in table 2 .

Table 2. Purposes of taking fitness gymnastics courses

(the total number is 400)

\begin{tabular}{lcc}
\hline \multicolumn{1}{c}{ Purpose } & Number & Proportion \\
\hline Personal interests & 380 & $95 \%$ \\
Getting credits & 100 & $25 \%$ \\
Getting fitter & 260 & $65 \%$ \\
Learning fitness methods & 366 & $91.5 \%$ \\
Mastering sports skills & 153 & $38.2 \%$ \\
Improving comprehensive quality and & & \\
Learning related culture & 286 & $71.5 \%$ \\
Social Communication & 303 & $75.7 \%$ \\
Work demands in the future & 112 & $28 \%$ \\
Entertainment and the others & 163 & $40.7 \%$ \\
\hline
\end{tabular}


College students have all-round requirements for fitness gymnastics classes. When choosing purposes of physical training, 95\% students express that it's their personal interests; $91.5 \%$ students want to learn fitness methods; $75.7 \%$ students want to communicate with the others; only $38.2 \%$ of them want to master sports skills (Table 2). It can be seen that college students have all-round requirements for fitness gymnastics classes. They want to communicate with others, and present themselves through sports. Fitness gymnastics has entertainment functions and aesthetic values, and can enrich students' lives. Therefore, it becomes an irresistible temptation for the majority of young students.

College students' requirements of physical activities advance with times. As we can see from table 2, $28 \%$ students choose the course to meet their work demands in the future. $40.7 \%$ of students take the course for entertainment or other reasons. It shows that nowadays, some sports activities cannot fully meet the needs of students; young students in the new era need sports activities which have new content and new characteristics. With the emergence of the national sports fervor, more and more people are engaging in fitness gymnastics. As a useful activity, fitness gymnastics can help people to achieve the best psychological states in beautiful melodies, and enrich their lives in spare time. So, learning fitness gymnastics is good for social communication. After mastering certain fitness gymnastics knowledge in schools, students can better adapt to the society after graduation. At the same time, it can also promote the development of national fitness project, and brings lifelong benefits to citizens.

College students attach great importance to external beauty. From table 2, we can see that $65 \%$ students want to get fitter through fitness gymnastics courses. Based on their interests, students want to have graceful and muscular bodies. They hope that through fitness gymnastics exercises, they can become fitter, more vigorous and have more gentle movements. They desire to be beautiful and admired by others. At the same time, fitness gymnastics is a rigorous physical activity which can enrich campus culture. As a kind of collective activity, it can intensify communication and promote friendship between students.

Teachers' qualifications and attitudes. Teachers' qualifications

Table 3 Teachers' qualifications in private higher learning institutions in Changchun (the total number is 40)

\begin{tabular}{lcc}
\hline & Number & Proportion \\
\hline Gymnastics & 5 & $12.5 \%$ \\
Aerobic & 10 & $25 \%$ \\
Others & 25 & $62.5 \%$
\end{tabular}

According to the survey, in private higher learning institutions in Changchun, 12.5\% teachers are professional in gymnastics, $25 \%$ are professional in aerobics. The data shows that teacher resources on fitness gymnastics courses are relatively weak.

Teachers' attitudes towards fitness gymnastics. Through the survey, we found that in these schools, teachers, especially gymnastics and aerobics teachers, show great interests in fitness gymnastics courses. They are willing to teach these classes. 60\% teachers from other majors are also very interested in fitness gymnastics courses; they even try to learn how to teach these classes.

\section{Conclusions and Suggestions}

Conclusions. Fitness gymnastics can not only promote students' physical and mental health, but also improve their cultural and artistic accomplishments. It can satisfy the interests of contemporary college students, and fit in with the psychological and physiological characteristics of them.

Fitness gymnastics courses should get rid of discipline-based ideas, and pay attention to students' requirements and social demands. The courses need to attach importance to the cultivation of 
students' awareness of lifelong sports and health protection, and promote the all-round development of students. These are the overall guiding principles of fitness gymnastics courses.

Suggestions. All sports are dangerous to some extent. The course should not be prohibited because of safety risk factors. Teachers should maintain high senses of responsibility, and always put safety first.

Establishing fitness gymnastics clubs. Students cannot learn everything in the classroom. Clubs provide them with opportunities of further training and learning. Through the establishment of these clubs, the influence of fitness gymnastics can be enhanced in private higher learning institutions, which is good for the development of fitness gymnastics courses.

Strengthening communications with other private higher learning institutions, as well as sports colleges and universities. Then, the development orientation of setting fitness gymnastics courses can be explored together. Curriculum content system can also be improved in that way. Fitness gymnastics courses with their own characteristics should be offered according to the requirements of students and the social development. Then the course selection system can be optimized. Specific strategies include using modern teaching methods, increasing financial input and reforming evaluation system. Summative assessment should be combined with process assessment. Teachers need to promote the development of students' comprehensive qualities and social adaptation abilities, and cultivate their awareness of lifelong sports and maintaining healthy lifestyle.

\section{References}

[1] P.G. Zhang, Gymnastics Education, Changchun Publishing House, Changchun, 2007:1.

[2] B.S. Cheng, X.H. Liu, Fitness Gymnastics: Five-animal Exercises, Chemical Industry Press, Beijing, 2007. p1.

[3] Z. Mo, Research on challenges and corresponding reform measures of traditional gymnastics teaching, J. Contemporary Sports Technology. 28 (2013) 68-69.

[4] X.L. Lu, K. Wang, Research on current teaching modes of gymnastics, J. Journal of Jilin Province Economic Management Cadre College. 6 (2015) 76-78.

[5] L. Yang, Optimization analysis on the contents and curriculum objectives of physical education in colleges and universities, J. Science and Technology of Stationary and Sporting Goods. 6 (2015) 66-67.

[6] Q. Chen, Thinking of deepening the reform of physical education in private higher learning institutions: a brief discussion on the reform of physical education in Xiamen Xingcai Polytechnic, J. Science and Technology Information. 18 (2010) 195

[7] W. Zhou, Research on the reform of professional gymnastics compulsory courses in physical education institutes and departments in China - constructing the basic framework of fitness gymnastics course content, J. Journal of Beijing Sport University. 30 (2007) 822-824.

[8] J.S. Xu, Characteristics of vocational education in Australia and enlightenment, J. Vocational and Technical Education. 2009

[9] X.D. Jiang, Educational supermarket: construction and pursuit of an ideal education, J. Journal of Tianjin Normal University. 9 (2008) 40-44. 